\title{
Use of Zoledronic Acid in a Neonate with Subcutaneous Fat Necrosis Complicated with Severe, Refractory Hypercalcemia
}

\author{
Maria Angela Militello, MD ${ }^{1}$ Maria Paola Re, MD ${ }^{2}$ Giuliana Vitaliti, MD ${ }^{3}$ Francesca Finazzo, MD ${ }^{2}$ \\ Paolo Manzoni, MD ${ }^{1}$ Salvino Marcello Vitaliti, MD ${ }^{2}$
}

${ }^{1}$ Division of Pediatrics, Department of Maternal, Neonatal, and Infant Medicine, Nuovo Ospedale degli Infermi, Biella, Italy

2 U.O.C. Neonatologia - TIN - Nido, ARNAS Civico, Palermo, Italy

3 Dipartimento di Scienze per la Promozione della Salute e Materno Infantile G. D'Alessandro, University of Palermo, School of Medicine, Palermo, Italy

Am J Perinatol 2019;36(suppl S2):S134-S138.

\begin{abstract}
Address for correspondence Maria Angela Militello, MD, Division of Pediatrics, Department of Maternal, Neonatal, and Infant Medicine, Nuovo Ospedale degli Infermi, Via dei Ponderanesi, 2-13058, Ponderano, Biella, Italy (e-mail: mariangela.militello@aslbi.piemonte.it).
\end{abstract}

\begin{abstract}
Keywords

- hypercalcemia

- subcutaneous fat necrosis

- zoledronic acid

- neonate

Objective Subcutaneous fat necrosis (SCFN) is a rare condition that may occur in the neonatal period. SCFN is an inflammatory disorder of the adipose tissue, usually found in full-term healthy infants who have a history of intrauterine or perinatal distress. It is usually a self-limited condition; however, in some cases, it can get complicated, leading to severe hypercalcemia that may be life-threatening.

Study Design We report and describe a classic presentation of SCFN that led to severe hypercalcemia refractory to standard treatment. The diagnosis of SCFN was made based on the finding of subcutaneous nodules and of hypercalcemia. The serum calcium level reached $16.6 \mathrm{mg} / \mathrm{dL}$. Hypercalcemia was treated first with intravenous infusions of fluids and furosemide and then of methylprednisolone. This standard treatment was not effective; therefore, we administered a single low dose of zoledronic acid, which, in turn, was efficacious in ultimately managing the hypercalcemia.

Conclusion Our case shows how a single low dose of zoledronic acid was safe and effective in managing severe hypercalcemia unresponsive to conventional treatment while minimizing the risk of hypocalcemic rebounds.
\end{abstract}

Subcutaneous fat necrosis (SCFN) of neonates is a rare condition whose exact incidence is unknown, affecting infants born at term or postterm in the very first weeks of life. Major clinical features include firm, circumscribed, subcutaneous, flesh-colored-to-blue nodules and plaques on the trunk, buttocks, thighs, arms, and cheeks. Prognosis is good, with complete regression within a few weeks. The main acute complications are dyslipidemia and thrombopenia, as well as hypercalcemia that may be severe and lifethreatening. The pathophysiology of SCFN remains unknown. The most common hypothesis is that systemic or local conditions might induce subcutaneous hypoxemia and adipocyte necrosis. Predisposing factors for this condition include maternal diseases (such as gestational diabetes, preeclampsia, and exposure to cocaine or calcium blocker during pregnancy) and neonatal conditions (such as hypothermia, infections, hypoxemia, cutaneous trauma, cord accidents, meconium aspiration, anemia, and thrombocytosis). ${ }^{1,2}$

\section{Case Presentation and Treatment}

We describe a classic presentation of SCFN with hypercalcemia refractory to standard treatment. Our patient was a full-term female infant, adequate for gestational age, born through emergency cesarean section due to fetal bradycardia. Her Apgar scores at 1,5 , and 10 minutes were 5,7 , and 9 respectively. The baby at birth required ventilatory support
Copyright $\odot 2019$ by Thieme Medical Publishers, Inc., 333 Seventh Avenue, New York, NY 10001, USA. Tel: +1(212) 584-4662. ISSN 0735-1631. 
Table 1 Overview of the major laboratory exams results at admission and at discharge

\begin{tabular}{|c|c|c|c|}
\hline \multirow[t]{2}{*}{ Analyte } & \multicolumn{2}{|l|}{ Results } & \multirow{2}{*}{$\begin{array}{l}\text { Reference } \\
\text { range } \\
\text { (neonatal } \\
\text { age) }\end{array}$} \\
\hline & Admission & Discharge & \\
\hline Calcium, mg/dL & 16.6 & 10.1 & $8.6-10.2$ \\
\hline Phosphate, mg/dL & 5.0 & 4.3 & $4.5-6.7$ \\
\hline $\begin{array}{l}\text { Parathyroid } \\
\text { hormone, } \mathrm{pg} / \mathrm{mL}\end{array}$ & 3.11 & & $10-65$ \\
\hline $\begin{array}{l}\text { Alkaline } \\
\text { phosphatase, IU/L }\end{array}$ & 151 & & $100-400$ \\
\hline $\begin{array}{l}\text { 25-Hydroxyvitamin } \mathrm{D} \text {, } \\
\mathrm{ng} / \mathrm{mL}\end{array}$ & 50.4 & 36.8 & $20-100$ \\
\hline Creatinine,mg/dL & 0.6 & 0.4 & $0.2-0.4$ \\
\hline Urea, mg/dL & 14 & 9 & $5-25$ \\
\hline $\begin{array}{l}\text { Urine calcium: } \\
\text { creatinine ratio }\end{array}$ & 2.6 & 0.85 & $<0.86$ \\
\hline
\end{tabular}

and was referred to the local NICU. She was hypotensive and anemic (Ht 20\%), requiring intravenous (IV) bolus administration of fluids and inotropes (dopamine) as well as red blood cell transfusion.

When she was 3 weeks old, she was readmitted for failure to thrive and hyporexia. On admission, the infant weighed 3,030 g (3rd percentile), and her length and her head circumference were also at the 10th percentile. Her vital signs were normal; her examination revealed hypotonia and lethargy. Laboratory tests are presented in -Tables $\mathbf{1}$ and 2. Notably, the serum calcium level was $16.6 \mathrm{mg} / \mathrm{dL}(8.6-10.2 \mathrm{mg} / \mathrm{dL})$. A renal ultrasound disclosed dense medullary nephrocalcinosis (-Fig. 1).

During the second week of hospitalization, the infant developed indurated, erythematous plaques on her left

Table 2 Daily trends of calcium serum and urine levels

\begin{tabular}{|l|l|l|}
\hline $\begin{array}{l}\text { Weeks } \\
\text { of life }\end{array}$ & $\begin{array}{l}\text { Serum calcium, } \\
8.6-10.2 \mathrm{mg} / \mathrm{dL}\end{array}$ & $\begin{array}{l}\text { Urine calcium: } \\
\text { creatinine ratio } \\
\mathbf{0} .86\end{array}$ \\
\hline 3.5 & 16.6 & \\
\hline 3.6 & 15.4 & \\
\hline 4.1 & 13.7 & 1.03 \\
\hline 4.2 & & 2.61 \\
\hline 4.3 & & \\
\hline 4.4 & 11.0 & 1.13 \\
\hline 5.2 & & 2.1 \\
\hline 5.4 & 12.4 & \\
\hline 5.5 & 13.4 & \\
\hline 5.6 & 14.4 & 1.49 \\
\hline 6.0 & 15.1 & \\
\hline 6.1 & 11.6 & 0.85 \\
\hline 6.2 & 9.5 & \\
\hline
\end{tabular}
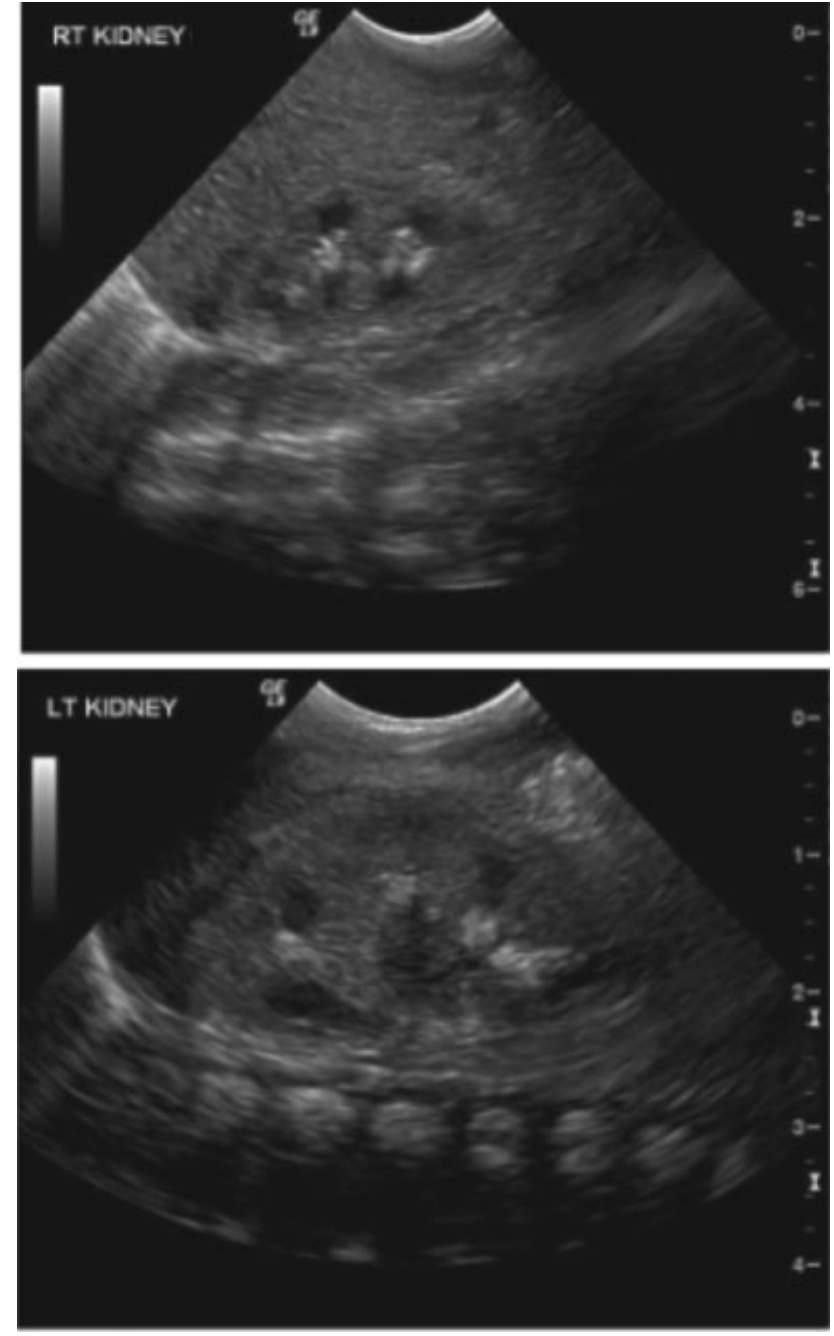

Fig. 1 Bilateral medullary nephrocalcinosis (renal ultrasound).

arm, back, and cheeks. Plaque's size varied from several millimeters to $2 \mathrm{~cm}$ (-Figs. 2 and $\mathbf{3}$ ).

A diagnosis of SCFN was made based on hypercalcemia and the finding of subcutaneous nodules.

Hypercalcemia was managed with intravenous infusions of fluids and treatment with furosemide, leading to transient normalization of calcium levels. Despite the therapy, on the second week of hospitalization, total calcium was elevated $(14.4 \mathrm{mg} / \mathrm{dL})$. Treatment with methylprednisolone (IV) ( $4 \mathrm{mg} / \mathrm{kg} / 24$ hour) was then started, but the calcium level increased $(15.1 \mathrm{mg} / \mathrm{dL})$; therefore, we administered a single low dose of zoledronic acid $(0.025 \mathrm{mg} / \mathrm{kg}$ single dose) (-Fig. 4). Subsequently, the patient's calcium level rapidly decreased from 15 to $9.5 \mathrm{mg} / \mathrm{dL}$. IV fluid therapy was stopped and methylprednisolone was tapered over the next week.

The clinical patient's conditions normalized; the subcutaneous nodules progressively decreased in size and then completely resolved at discharge. The renal findings suggestive of nephrocalcinosis improved over time.

Despite that she was fed a standard infant formula, no other zoledronic acid administration was needed. She was discharged on the seventh week of life with regular follow-up. 


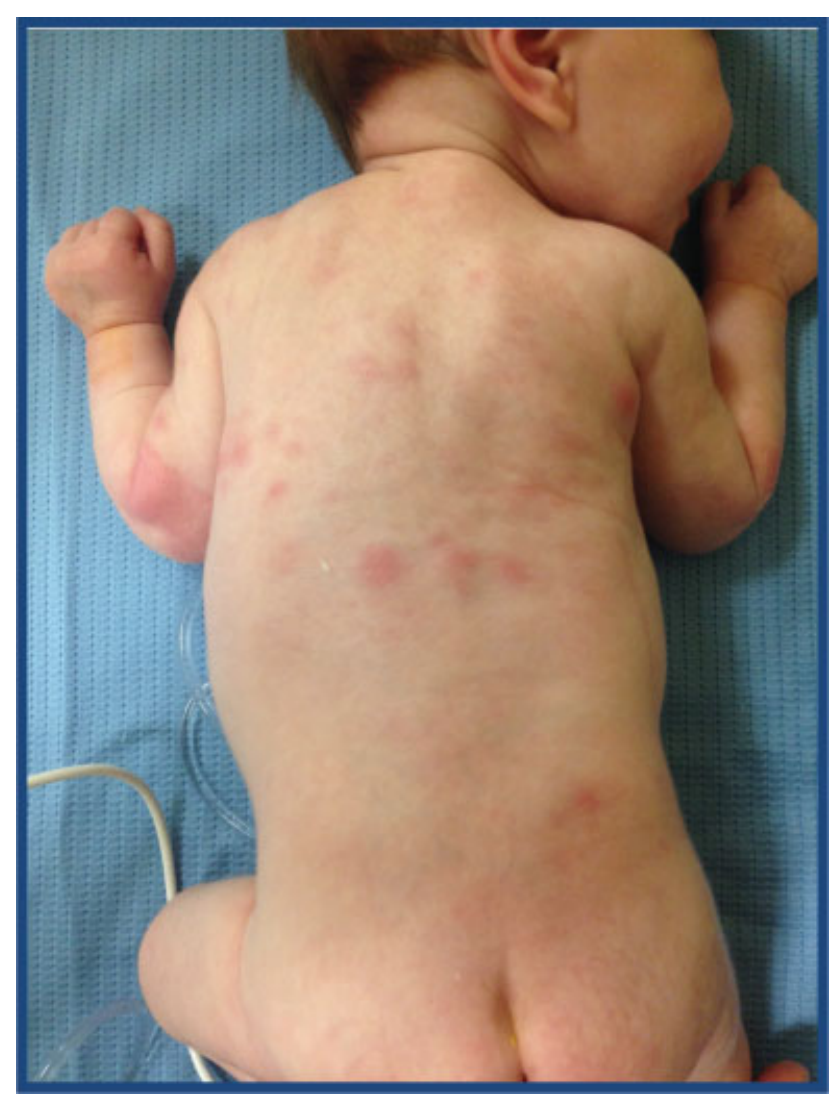

Fig. 2 A 3-week-old infant with lesions of subcutaneous fat necrosis located on the left arm, back, and cheeks.

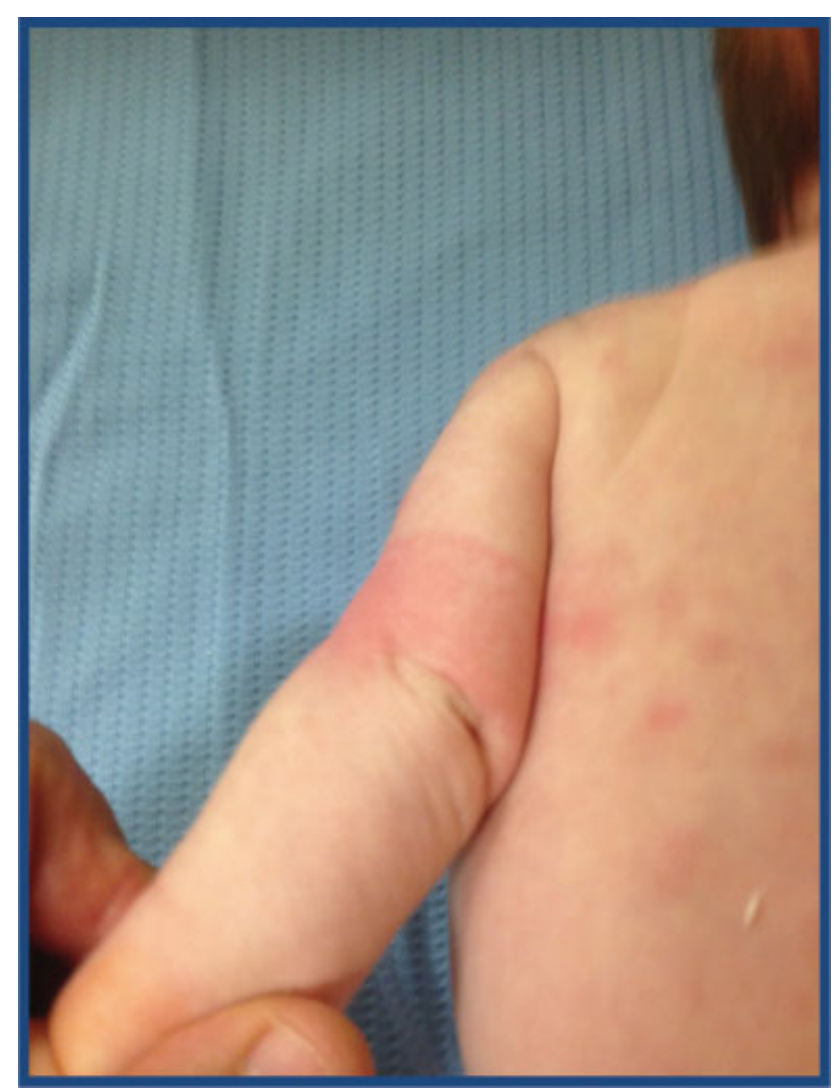

Fig. 3 Subcutaneous fat necrosis on the left arm of the same infant.

\section{Serum Calcium $\mathrm{mg} / \mathrm{dL}$}

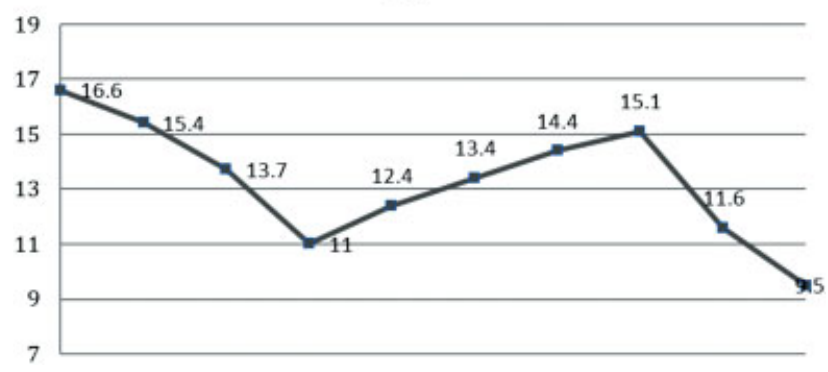

Fig. 4 Serum calcium levels and treatment.

\section{Discussion}

SCFN is an inflammatory disorder of adipose tissue, usually occurring in full-term healthy infants who have a history of intrauterine or perinatal distress. Ischemic injury, hypoxia, or hypothermia can damage the subcutaneous adipose tissue, causing fat to crystallize in necrotizing granulomas. SCFN is characterized by multiple indurated plaques or nodules with or without erythema on the cheeks, buttocks, posterior trunk, and extremities, which develop within the first weeks of life. SCFN usually is a self-limiting process that progresses toward resolution in a period ranging from few weeks to 6 months, with lesions evolving into fibrotic scars with fat atrophy. This disease very rarely leads to cutaneous sequelae; however, complications can be severe and require pharmacological treatment. ${ }^{3}$ The main clinical differential diagnosis is with sclerema neonatorum, which appears in preterm, ill infants as a diffuse, white and yellowish, woody induration that rapidly involves almost entirely the body surface except the palms, soles, and genitals, and yields poor prognosis. ${ }^{4}$ Erythema nodosum also has to be considered in the differential diagnosis; this is a septal panniculitis, with little fat necrosis of the lobules and no crystals. ${ }^{3}$

Although SCFN of the newborn is often benign and selflimiting, the most important complication is hypercalcemia (defined as total serum calcium $>10.8 \mathrm{mg} / \mathrm{dL}$ or ionized calcium $>5.4 \mathrm{mg} / \mathrm{dL}$ ), which can lead to neurologic or cardiac problems, nephrocalcinosis, and nephrolithiasis. ${ }^{5}$ Typically, hypercalcemic neonates are asymptomatic, but very high calcium levels ( $>12,8 \mathrm{mg} / \mathrm{dL}$ ) can cause lethargy, irritability, hypotonia, constipation, vomiting, poor feeding, failure to thrive, and hypertension. ${ }^{6}$ Hypercalcemia can significantly reduce urinary concentrating capacity, producing polyuria; renal failure and dehydration may also occur. Hypercalcemia may be fatal if unrecognized, with the development of seizures and ultimately cardiac arrest. ${ }^{3,6}$

In neonates, severe hypercalcemia occurs rarely and may be determined by iatrogenic vitamin $\mathrm{D}$ intoxication or excessive calcium supplementation, granulomatous disease, SCFN of the newborn, Williams' syndrome, and hyperparathyroidism.

Laboratory work-up for neonatal hypercalcemia should include phosphate, alkaline phosphatase, 25-hydroxyvitamin $\mathrm{D}$, and parathyroid hormone measurement. If the diagnosis remains unclear, blood samples for 1,25- 
dihydroxyvitamin D and DNA analysis (calcium-sensing receptor and elastin genes) should be stored for later testing if necessary. Urine calcium, phosphate, and creatinine levels can help determine whether the kidneys are inappropriately retaining calcium, and a renal ultrasound should be performed if hypercalcemia is persistent and severe. ${ }^{6}$ Infants with SCFN typically have low parathormone levels with elevated 1,25-hydroxyvitamin D levels, but 1,25hydroxyvitamin D levels may occasionally be normal. Granulomatous lesions, similar to sarcoidosis, can increase 1hydroxylase activity, with increased extrarenal production of 1,25-hydroxyvitamin D. Local inflammation may increase production of bone-resorbing prostaglandin E, which, in turn, may lead to osteoclast activation. ${ }^{7}$ Skin lesions typically resolve over a period of weeks to several months; however, hypercalcemia can persist longer and requires continuous monitoring.

Management of neonatal hypercalcemia spans from conservative measures, such as IV fluid therapy and restriction of vitamin D and calcium, to more aggressive interventions, such as administration of furosemide, glucocorticoid, or bisphosphonate in severe cases. ${ }^{5}$

Currently, the evidence in literature is scarce and does not allow to perform any meta-analysis in this area. There is a lack of evidence coming from randomized controlled studies, as recently pointed out in a comprehensive review that included only case series and case reports. ${ }^{8}$

Several studies have reported so far the use of bisphosphonate products to manage severe neonatal hypercalcemia. Use of pamidronate was reported by Samedi et al in two infants with severe hypercalcemia associated with SCFN following therapeutic hypothermia for hypoxic-ischemic encephalopathy. ${ }^{9}$ Alos et al described four newborns presenting in the years 2001 to 2004 with severe hypercalcemia complicating SCFN. These patients were given three to four doses $(0.25-0.50 \mathrm{mg} / \mathrm{kg} /$ dose $)$ of pamidronate following unsuccessful treatment with IV fluids, low calcium diet, and furosemide. Urinary calcium/creatinine ratios and calcium levels decreased within 48 to 96 hours. 1,25-dihydroxyvitamin D levels normalized with the resolution of the skin lesions, and no persistent nephrocalcinosis was observed. ${ }^{10}$ Pérez Martínez et al provided further evidence of etidronate as an alternative and effective treatment for moderate-tosevere hypercalcemia. ${ }^{11}$ Trullemans et al reported the case of a patient presenting with symptomatic hypercalcemia complicated by nephrocalcinosis consecutive to SCFN after birth asphyxia at term. Oral etidronate was then used for 3 weeks after failure of standard treatment, and the evolution was favorable. ${ }^{12}$ As for safety, it is worthy to mention that hypersensitivity reactions to etidronate (rashes) have been occasionally described. ${ }^{13}$

Zoledronic acid is a third-generation bisphosphonate with a 100- to 200-fold higher potency than pamidronate. Similar to other bisphosphonate products, zoledronic acid inhibits osteoclast function and thus bone resorption. With its short infusion time and the need for infrequent infusions, zoledronic acid has potential advantages over pamidronate in the management of pediatric disease. It has been shown that reductions of the starting dose of zoledronic acid may result in decreased incidence of postinfusion, rebound hypocalcemia, thus improving safety. In addition, dosages tailored to the body mass index or body surface area, instead of body weight, may further reduce the incidence of rebound hypocalcemia. ${ }^{14}$ In the literature, only scattered experiences have been reported so far on the use of zoledronic acid in neonates. Di Bari et al treated an infant affected by SCFN and whose serum calcium levels were extremely high (15 $\mathrm{mg} / \mathrm{dL}$ ) with prednisolone and low-dose zoledronic acid. Serum calcium promptly normalized without rebound hypocalcemia, and redosing of zoledronic acid was not necessary. $^{7}$

Our report deserves attention for several reasons. First, we were able to manage very high serum calcium levels that could have been fatal since the routine standard treatments had not been effective. Second, we report on a novel use of a third-generation bisphosphonate that proved to be rapidly effective with a schedule comprising only a single low dose, with overall good tolerability. We acknowledge some limitations of this study, with the main one being that ours is a case report study from a single center. Since SCFN-related severe neonatal hypercalcemia is a rare condition, it seems difficult to get a numerically significant sample to conduct a study providing higher levels of evidence.

\section{Conclusion}

Severe neonatal hypercalcemia following SCFN requires a prompt identification and an effective treatment to prevent serious complications and death. Our case shows how a single, low dose of zoledronic acid was effective in treating severe hypercalcemia unresponsive to conventional treatments while minimizing the risk of rebound hypocalcemia.

\section{Funding \\ None.}

Conflict of Interest

None declared.

\section{References}

1 Mahé E, Girszyn N, Hadj-Rabia S, Bodemer C, Hamel-Teillac D, De Prost Y. Subcutaneous fat necrosis of the newborn: a systematic evaluation of risk factors, clinical manifestations, complications and outcome of 16 children. Br J Dermatol 2007;156(04):709-715

2 Burden AD, Krafchik BR. Subcutaneous fat necrosis of the newborn: a review of 11 cases. Pediatr Dermatol 1999;16(05):384-387

3 Lombardi G, Cabano R, Bollani L, Del Forno C, Stronati M. Effectiveness of pamidronate in severe neonatal hypercalcemia caused by subcutaneous fat necrosis: a case report. Eur J Pediatr 2009; 168(05):625-627

4 Tran JT, Sheth AP. Complications of subcutaneous fat necrosis of the newborn: a case report and review of the literature. Pediatr Dermatol 2003;20(03):257-261

5 Aljaser F, Weinstein M. A 1-week-old newborn with hypercalcemia and palpable nodules: subcutaneous fat necrosis. CMAJ 2008; 178(13):1653-1654

6 Tuddenham E, Kumar A, Tarn A. Subcutaneous fat necrosis causing neonatal hypercalcaemia. BMJ Case Rep 2015;2015:bcr2014208460 
7 Di Bari JA, Nead JA, Schurman SJ. Zoledronic acid for neonatal subcutaneous fat necrosis. Clinical Case Reports 2017;5(05): 567-569

8 Stefanko NS, Drolet BA. Subcutaneous fat necrosis of the newborn and associated hypercalcemia: A systematic review of the literature. Pediatr Dermatol 2019;36(01):24-30

9 Samedi VM, Yusuf K, Yee W, Obaid H, Al Awad EH. Neonatal hypercalcemia secondary to subcutaneous fat necrosis successfully treated with pamidronate: a case series and literature review. AJP Rep 2014;4(02):e93-e96

10 Alos N, Eugène D, Fillion M, Powell J, Kokta V, Chabot G. Pamidronate: Treatment for severe hypercalcemia in neonatal subcutaneous fat necrosis. Horm Res 2006;65(06):289-294
11 Pérez Martínez E, Camprubí Camprubí M, Ramos Cebrián M, et al. Treatment with bisphosphonates in severe hypercalcemia due to subcutaneous fat necrosis in an infant with hypoxic-ischemic encephalopathy. J Perinatol 2014;34(06):492-493

12 Trullemans B, Bottu J, Van Nieuwenhuyse J-P. Etidronate per os in subcutaneous fat necrosis with hypercalcemia and nephrocalcinosis [in French]. Arch Pediatr 2007;14(02):170-172

13 Rice AM, Rivkees SA. Etidronate therapy for hypercalcemia in subcutaneous fat necrosis of the newborn. J Pediatr 1999;134 (03):349-351

14 Munns CF, Rajab MH, Hong J, et al. Acute phase response and mineral status following low dose intravenous zoledronic acid in children. Bone 2007;41(03):366-370 